\title{
Chlorthalidone Plus Amiloride Reduces the Central Systolic Blood Pressure in Stage 1 Hypertension Patients
}

\author{
Leticia Aparecida Barufi Fernandes ${ }^{\mathrm{a}}$, Elizabeth do Espirito Santo Cestario ${ }^{\mathrm{a}}$, \\ Luciana Neves Cosenso-Martin ${ }^{\mathrm{b}}$, Jose Fernando Vilela-Martin ${ }^{\mathrm{a}}$, \\ Juan Carlos Yugar-Toledo a, d, Flavio Danni Fuchs ${ }^{\mathrm{c}}$
}

\begin{abstract}
Background: Hypertension reduction strategies use blood pressure in the brachial artery as the primary endpoint. Individuals who achieve the target blood pressure reduction with antihypertensive treatment have residual cardiovascular risk attributed to the difference in pressure between the aorta and brachial artery. Antihypertensive treatment affects the intrinsic properties of the vascular wall and arterial stiffness markers and consequently the central pressure. Recent publications stress the importance of adequate control of the central compared to peripheral blood pressure. Related clinical implications suggest that individuals with normal peripheral but high central blood pressure should not receive antihypertensive drugs that act on the central pressure. Therefore, they are at greater cardiovascular risk. The aim of the study was to evaluate the effect of treatment with a thiazide diuretic versus losartan on the central blood pressure in stage 1 hypertensive patients.
\end{abstract}

Methods: Twenty-five patients were randomized to the chlorthalidone $25 \mathrm{mg}$ /amiloride $5 \mathrm{mg}$ group (q.d.) and 25 patients received losartan $50 \mathrm{mg}$ (b.i.d). The central systolic blood pressure (CSBP) and augmentation index (AIx 75) were assessed using applanation tonometry. The paired $t$-test was used to compare the systolic blood pressure (SBP), diastolic blood pressure (DBP), mean arterial pressure (MAP), pulse pressure (PP), CSBP and AIx 75 between the thiazide and losartan groups at baseline and after 6 months of treatment.

Results: Significant reductions in CSBP (123.3 \pm 14.2 vs. $113.4 \pm$ $111.4, \mathrm{P}=0.0103)$ and AIx $75(87.7 \pm 9.6$ vs. $83.8 \pm 8.9, \mathrm{P}=0.0289)$ were observed after 6 months of drug treatment with chlorthalidone $25 \mathrm{mg} /$ amiloride $5 \mathrm{mg}$ (q.d.). The administration of losartan $50 \mathrm{mg}$ (b.i.d) did not reduce the CSBP and there were insignificant changes

Manuscript accepted for publication September 30, 2016

aHypertension Clinic, Department of Internal Medicine, State Medical School of Sao Jose do Rio Preto (FAMERP), Sao Paulo, Brazil

${ }^{b}$ Endocrinology Division of the Internal Medicine Department, State Medical School of Sao Jose Rio Preto, SP (FAMERP), Brazil

'Division of Cardiology, Hospital de Clinicas de Porto Alegre, Universidade Federal do Rio Grande do Sul, Porto Alegre, Rio Grande do Sul, Brazil ${ }^{\mathrm{d} C}$ Corresponding Author: Juan Carlos Yugar-Toledo, Las Vegas 200, Sao Jose do Rio Preto/SP, CEP 15093-010, Brazil. Email: yugarjuan@uol.com.br

doi: https://doi.org/10.14740/cr481w in the AIx 75.

Conclusions: Six-month treatment of chlorthalidone/amiloride but not losartan reduces the CSBP and AIx 75 in adults with stage 1 hypertension.

Keywords: Stage 1 hypertension; Central systolic blood pressure; Chlorthalidone; Amiloride; Losartan

\section{Introduction}

Hypertension (HTN) is a major risk factor for cardiovascular diseases such as stroke (mostly hemorrhagic), coronary heart disease (acute myocardial infarction), left ventricular hypertrophy, congestive heart failure, and aortic dissection, as well as kidney failure and peripheral vascular disease $[1,2]$. HTN is defined when the systolic blood pressure (SBP) is greater than $140 \mathrm{~mm} \mathrm{Hg}$ or the diastolic blood pressure (DBP) is greater than $90 \mathrm{~mm} \mathrm{Hg} \mathrm{[3].}$ The mortality rate due to cardiovascular diseases progressively increases in a continuous independent linear manner in adults as the blood pressure (BP) increases from 115/75 $\mathrm{mm} \mathrm{Hg}$ [3].

The aorta, a vessel with dynamic characteristics that maintains a certain pressure coefficient known as the central systolic blood pressure (CSBP), has endocrine, autocrine and paracrine functions, and is capable of undergoing rapid changes in its morphology due to its elasticity. This is mediated by highly complex mechanisms of regulation controlled by hemodynamic factors, local and systemic responses, neurohumoral activation, and changes in vascular reactivity that can be genetically determined and that can eventually lead to changes in the structure and function of the arterial wall, thereby stimulating the development of arterial stiffness $[4,5]$.

Arterial stiffness is a major determinant of increased pulse pressure (PP) and CSBP. Higher cardiovascular morbidity and mortality have been associated with increased CSBP [6], especially in diabetic patients and the elderly, and associated with chronic nephropathy $[7,8]$. It is estimated that the arteries, especially the aorta and carotid, stiffen from $10 \%$ to $15 \%$ each decade in men and from $5 \%$ to $10 \%$ in women $[9,10]$.

Several techniques have been extensively used to measure the increase in arterial stiffness, such as the evaluation of pulse wave velocity (PWV) and estimates of CSBP by non-invasive 


\section{Stage 1 Hypertension Group (HTN-1)}

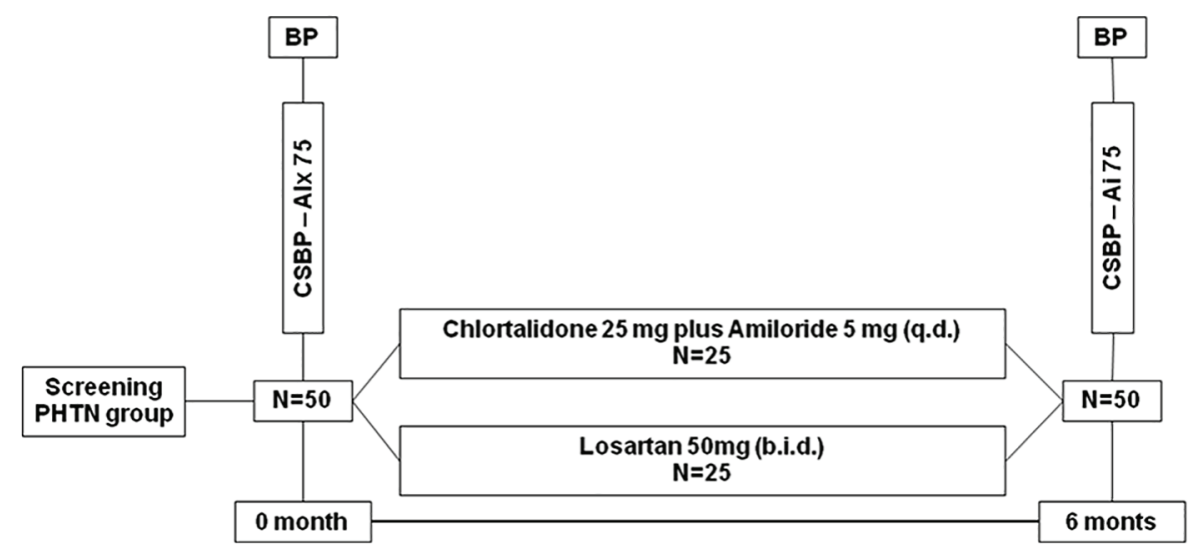

Figure 1. Research design. CSBP: central systolic blood pressure; Alx 75: augmentation index 75 beats/min.

methods $[11,12]$. The combined changes of the forward (ejection) and backward waves (peripheral reflection) that alter the pulse wave profile can be recorded non-invasively by applanation tonometry (AT) of the radial artery $[13,14]$.

This method provides information about the functional condition of the arterial bed and allows an analysis of the AIx, which is a calculation of the ratio between the reflected wave and the ejection wave. Hence, increased cardiovascular risk can be predicted early, that is, the AIx functions as a highly reliable marker of cardiovascular risk [15-17].

Antihypertensive treatment exerts distinct effects on the intrinsic properties of the vascular wall and arterial stiffness markers and hence on the CSBP. Recent publications emphasize the importance of adequate control of CSBP compared to peripheral BP [18], and these authors suggest that the CSBP is an independent risk factor for the development of brain and cardiovascular diseases [19].

The objective of this study was to evaluate the effect of treatment using low doses of thiazide diuretic chlorthalidone plus amiloride or angiotensin II receptor blockers on the CSBP and arterial stiffness in patients with stage 1 hypertension (HTN-1).

\section{Patients and Methods}

\section{Patients}

The project was approved by the Research Ethics Committee of Hospital de Base of the Medical School in Sao Jose do Rio Preto (FAMERP) (\#2205/2009). The nature of the study was carefully explained to patients and all individuals, after they agreed to participate in the study, signed informed consent forms and filled out a standard questionnaire. Fifty patients with HTN-1 of both genders (25 males and 25 females), aged between 40 and 70 years with medical diagnosis of arterial, were included in this randomized study. The study inclusion criteria were to keep regular scheduled medical appointments, and to be able to understand, verbalize and answer questions.

Patients with secondary hypertension, known cardiovascular diseases such as previous infarction, stroke, heart failure, or clinical manifestations including current or previous angina and poor circulation of the legs, or any chronic illness that limited their participation or life expectancy (e.g. cancer, disabling rheumatic disease) were excluded from the study. Moreover, patients taking long-term analgesic or anti-inflammatory drugs ( $\geq 30$ days), women who were pregnant or would possibly become pregnant during the study and patients who had participated in other studies within the previous 6 months were excluded.

All participants underwent physical examinations and investigations of cardiovascular disease in their family history. Initially data on age, gender, weight, and height, an electrocardiogram and results of biochemical blood tests were collected.

Oscillometric BP was taken using a Microlife device (3AC1-1 Widnau, Switzerland) as validated by the European Society of Hypertension using a cuff with an adequate arm circumference size and a width/length ration of 1:2. The average of three measurements was calculated for the SBP and DBP.

The CSBP and AIx were evaluated by a non-invasive technique, AT, using an Omrom HEM-9000AI tonometer (Omron Healthcare, Kyoto, Japan) and the AIx of the radial artery was calculated.

When the status of the patient was defined as HTN-1, the individual received guidance on necessary changes in lifestyle [20]. If there was no change in their BP by the end of 3 months, the patient was prescribed a single formulation of chlorthalidone $25 \mathrm{mg}$ with amiloride $5 \mathrm{mg}$ (q.d.), or losartan 50 $\mathrm{mg}$ (b.i.d.) for 6 months. At the end of 6 months of treatment, the CSBP and the AIx of the radial artery were again measured (Fig. 1).

AT

AT analyzes the $\mathrm{CBP}$ and BP simultaneously. Initially BP sta- 
Table 1. Demographic and Anthropometric Characteristics of the HTN-1 Patients

\begin{tabular}{lll} 
& Chlortalidone/amiloride & Losartan \\
\hline Age (years) & $54.6 \pm 7.1$ & $54.4 \pm 7.9$ \\
Gender: $\mathrm{F} / \mathrm{M}$ & $11 / 14$ & $16 / 9$ \\
Height $(\mathrm{m})$ & $1.64 \pm 0.1$ & $1.63 \pm 0.1$ \\
Weight $(\mathrm{kg})$ & $80.0 \pm 18.1$ & $80.0 \pm 18.3$ \\
BMI $\left(\mathrm{kg} / \mathrm{m}^{2}\right)$ & $29.5 \pm 5.9$ & $29.6 \pm 4.8$ \\
$\mathrm{~N}$ & 25 & 25 \\
\hline
\end{tabular}

Values are expressed as mean $\pm \mathrm{SD}$. ${ }^{*} \mathrm{P}<0.05$. Chlortalidone $25 \mathrm{mg} /$ amiloride $5 \mathrm{mg}$ (q.d.). Losartan $50 \mathrm{mg}$ (b.i.d.). HTN-1: stage 1 hypertension; BMI: body mass index.

bility is confirmed by checking that the difference between two readings does not exceed $10 \mathrm{~mm} \mathrm{Hg}$. Subsequently, the left forearm is placed on the support of the equipment, the tonometer sensor is placed on the radial artery and the AIx is calculated three times from BP measurements taken at 3-min intervals. The standard deviation of the three readings must not exceed $10 \%$ for the measurement to be considered valid. An AIx of less than $100 \%$ is considered normal. The more elastic the artery, the lower the percentage values of AIx [14, 21].

\section{Lipid and biochemical profiles}

Peripheral blood was collected from all participants to analyze the lipid and biochemical profiles. Serum triglycerides (TG), total cholesterol (TC) and the high-density lipoprotein cholesterol fraction (HDL-c) were determined by standard enzymatic colorimetric methods [22-24]. The low-density cholesterol fraction (LDL-c) was calculated using the Friedewald formula [25] such that LDL-c $(\mathrm{mg} / \mathrm{dL})=$ TC $(\mathrm{HDL}-\mathrm{C}+\mathrm{TG} / 5)$, except for TG values above $400 \mathrm{mg} / \mathrm{dL}$ when the LDL-c concentration was not calculated.

Serum levels of glucose, microalbuminuria, glycated hemoglobin, serum potassium, and uric acid were also measured and the renal function was assessed by the blood creatinine level and the estimated glomerular filtration rate (eGFR) [26].

\section{Statistical analysis}

Quantitative variables were expressed as mean \pm standard deviation. The analysis of paired $t$-test was used to compare the CSBP in HTN-1 patients at 0 and 6 months, and Chi-square test to compare proportions for qualitative variables. All statistical analyses were performed using the Sigmastat Statistical Software and Graph-Pad InStat version 5.0. The sample size was estimated based on an $80 \%$ power and the level of significance was set for $\mathrm{P}<0.05$.

\section{Results}

The mean age of the chlortalidone/amiloride group was $54.6 \pm$ 7.1 years, and the mean age of the losartan group was $54.4 \pm$ 7.9 years. Table 1 shows the demographic and anthropometric characteristics of the studied groups.

The mean baseline SBP and DBP were $143.4 \pm 3.0$ and $93.4 \pm 2.8 \mathrm{~mm} \mathrm{Hg}$, respectively in the chlortalidone/amiloride group. The mean baseline SBP and DBP were $143.3 \pm 4.5$ and $93.2 \pm 2.9 \mathrm{~mm} \mathrm{Hg}$, respectively in the losartan group. Variables including SBP, DBP, mean arterial pressure (MAP) and pulse pressure (PP) are listed in Table 2.

The results of biochemical tests are listed in Table 3.

The values of CSBP and AIx adjusted for a heart rate 75 beats/min (AIx 75) were compared at baseline and at 6 months of active treatment as shown in Table 4 and in Figure 2.

There were statistically significant reductions in the CSBP and AIx 75 in HTN-1 patients, when comparing the baseline with 6 months of treatment using chlorthalidone $25 \mathrm{mg}$ added to amiloride $5 \mathrm{mg}$ (q.d.), $\operatorname{CSBP}(123.3 \pm 14.2$ vs. $113.4 \pm 11.4$ $\mathrm{mm} \mathrm{Hg}, \mathrm{P}=0.0103)$ and AIx $75(87.7 \pm 9.6$ vs. $83.8 \pm 8.9, \mathrm{P}=$ $0.0289)$. However, no significant change was observed in the CSBP and AIx 75 after 6 months of treatment with losartan 50 mg (b.i.d.) (Fig. 3).

\section{Discussion}

The main results of this study show significant reductions in the AIx 75 and CSBP of patients with HTN-1 after 6 months of

Table 2. Characteristics of Hemodynamic HTN-1 Patients Before and After 6 Months of Treatment With Chlortalidone 25 mg/Amiloride $5 \mathrm{mg}$ (q.d.) or Losartan $50 \mathrm{mg}$ (b.i.d.)

\begin{tabular}{llllllll} 
& \multicolumn{3}{c}{ Chlortalidone/amiloride } & & \multicolumn{3}{c}{ Losartan } \\
\cline { 2 - 3 } & Baseline & $\mathbf{6 ~ m o n t h s}$ & P value & & Baseline & 6 months & P value \\
\hline SBP (mm Hg) & $143.4 \pm 3.0$ & $127.0 \pm 7.3$ & $<0.0001$ & & $143.3 \pm 4.5$ & $127.3 \pm 10.7$ & $<0.0001$ \\
DBP (mm Hg) & $93.4 \pm 2.8$ & $83.04 \pm 5.1$ & $<0.0001$ & & $93.2 \pm 2.9$ & $81.0 \pm 7.1$ & $<0.0001$ \\
MAP (mm Hg) & $110.2 \pm 2.4$ & $97.8 \pm 4.6$ & $<0.0001$ & & $109.9 \pm 2.1$ & $96.5 \pm 7.6$ & $<0.0001$ \\
PP (mm Hg) & $50.0 \pm 3.5$ & $43.9 \pm 7.9$ & 0.0007 & & $50.4 \pm 6.0$ & $46.3 \pm 7.8$ & 0.0584 \\
HR (bpm) & $77.1 \pm 12.8$ & $77.3 \pm 13.3$ & NS & & $71.8 \pm 11.0$ & $73.6 \pm 9.4$ & NS \\
N & 25 & & & 25 & & \\
\hline
\end{tabular}

Values are expressed as mean \pm SD. HTN-1: stage 1 hypertension; SBP: systolic blood pressure; DBP: diastolic blood pressure; MAP: mean arterial pressure; PP: pulse pressure; HR: heart rate. 
Table 3. Biochemical Values of HTN-1 Patients

\begin{tabular}{lll}
\hline & Chlortalidone/amiloride & Losartan \\
\hline Glycemia (mg/dL) & $132.9 \pm 93.85$ & $97.0 \pm 33.8^{*}$ \\
Glycated hemoglobin (mg/dL) & $7.02 \pm 2.37$ & $5.7 \pm 1.0^{*}$ \\
Total cholesterol (mg/dL) & $206.9 \pm 44.61$ & $217.1 \pm 33.6$ \\
LDL-cholesterol (mg/dL) & $119.7 \pm 34.92$ & $127.0 \pm 34.5$ \\
HDL-cholesterol (mg/dL) & $51.47 \pm 15.37$ & $49.6 \pm 14.0$ \\
Triglycerides (mg/dL) & $178.0 \pm 88.69$ & $201.6 \pm 93.5^{*}$ \\
Creatinine (mg/dL) & $0.95 \pm 0.22$ & $1.12 \pm 0.9$ \\
eGFR (MDRD) & $81.67 \pm 16.69$ & $78.3 \pm 28.1$ \\
Microalbuminuria (mg/24 h) & $14.67 \pm 11.60$ & $10.4 \pm 8.6$ \\
Potassium (mEq/L) & $4.04 \pm 0.49$ & $4.3 \pm 0.29$ \\
Uric acid (mg/dL) & $6.08 \pm 2.14$ & $5.9 \pm 1.3$ \\
N & 25 & 25 \\
\hline
\end{tabular}

Values are expressed as mean $\pm \mathrm{SD}$. ${ }^{*} \mathrm{P}<0.05$. Chlortalidone $25 \mathrm{mg} / \mathrm{amiloride} 5 \mathrm{mg}$ (q.d.). Losartan 50 mg (b.i.d.). HTN-1: stage 1 hypertension; LDL-c: low-density lipoprotein fraction; HDLc: high-density lipoprotein fraction; GFR-e: estimated glomerular filtration rate.

pharmacological treatment using chlorthalidone $25 \mathrm{mg}$ added to amiloride $5 \mathrm{mg}$ (q.d.) in contrast to losartan $50 \mathrm{mg}$ (b.i.d.) when no significant improvement was observed in respect to CSBP and AIx 75.

Some hypotheses can be suggested. Chlorthalidone has shown additional benefits beyond the reduction in BP when administered as monotherapy to patients with hypertension. These benefits include a reduction in oxidative stress, improved endothelial function, decreased vascular permeability and platelet aggregation, as well as stimulating angiogenesis which plays a key role in the restoration of arterial stiffness and vascular remodeling, components that are significantly involved in the genesis of elevated central pressure.

In contrast, some studies suggest that diuretics have no beneficial effect on central hemodynamics. This includes thiazide diuretics, which have been shown to have a neutral effect on reducing the central blood pressure and a neutral effect on the amplification of the PP. Consistent data are available for bendrofluazide and indapamide that also have a neutral effect on the AIx and PWV, respectively. However, although chlorthalidone has better antihypertensive efficacy than hydrochlorothiazide and preferably should be indicated for the treatment of hypertension, the literature is scarce in studies assessing this diuretic with respect to arterial stiffness and central pressure. Our results in HTN-1 patients contradict these claims.

It is known that aortic stiffness in the proximal segment is a consequence of greater backflow and that it occurs as part of aging process after the age of 60 . While, the stiffening of the aorta as a whole and of the great arteries such as the carotid and iliac arteries is related to an early wave reflection that promotes structural and functional changes of the vascular wall in these segments.

Therefore, the large arteries differ from medium and small caliber arteries in relation to histology, physiology and elastic properties, which is why the definition of the anatomical target for the action of a drug is extremely important. Evidence in relation to pharmacological treatment suggests that it is easier to revert changes to small caliber arteries (muscle) than to large

Table 4. Chlortalidone 25 mg/Amiloride 5 mg (q.d.) or Losartan 50 mg (b.i.d.), Central Systolic Blood Pressure, and Alx 75 of HTN-1 Group

\begin{tabular}{|c|c|c|c|c|c|c|}
\hline & \multicolumn{3}{|c|}{ Chlortalidone/amiloride } & \multicolumn{3}{|c|}{ Losartan } \\
\hline & Mean & SD & $95 \% \mathrm{CI}$ & Mean & SD & $95 \% \mathrm{CI}$ \\
\hline \multicolumn{7}{|l|}{ Baseline } \\
\hline $\mathrm{CBP}, \mathrm{mm} \mathrm{Hg}$ & 123.3 & 14.2 & $117.4-129.1$ & 120.5 & 16.7 & $113.6-127.4$ \\
\hline AIx 75 & 87.7 & 9.6 & $83.7-91.6$ & 91.2 & 8.7 & $87.7-94.9$ \\
\hline \multicolumn{7}{|l|}{6 months } \\
\hline $\mathrm{CBP}, \mathrm{mm} \mathrm{Hg}$ & $113.4^{*}$ & 11.4 & $108.9-118.2$ & 118.4 & 10.6 & $114.1-122.8$ \\
\hline AIx 75 & $83.8^{\#}$ & 8.9 & $80.1-87.4$ & 87.6 & 6.8 & $84.8-90.4$ \\
\hline
\end{tabular}

CSBP: central systolic blood pressure; Alx 75: augmentation index corrected for average heart rate of 75 bpm; HTN1: stage 1 hypertension. Values are expressed as mean \pm SD, Standard deviation; $95 \%$ Cl: 95\% confidence interval. ${ }^{*} \mathrm{P}=0.0103$. ${ }^{\mathrm{P}}=0.0289$. 


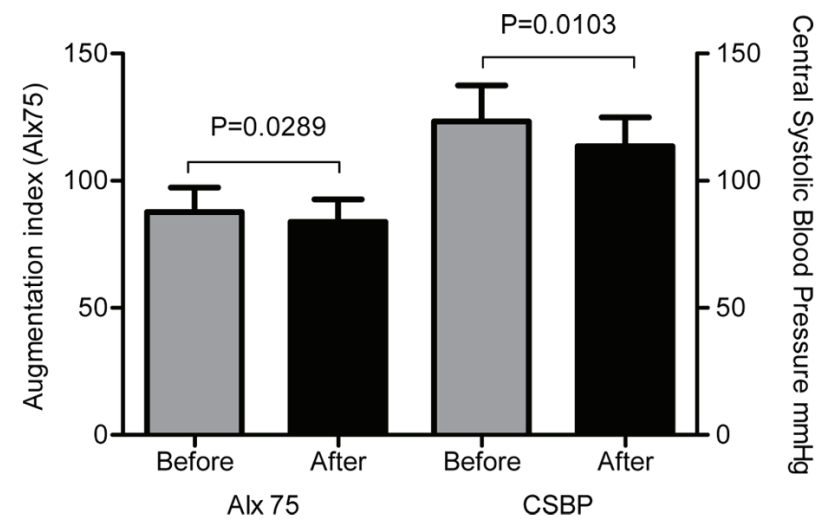

Figure 2. Central systolic blood pressure and augmentation index at before and after treatment with chlortalidone $25 \mathrm{mg}$ plus amiloride $5 \mathrm{mg}$ (q.d.) in the HTN-1 group. CSBP: central systolic blood pressure; Alx 75: augmentation index corrected for average heart rate of 75 beats/ min; HTN-1: stage 1 hypertension.

arteries (elastic). Thus, results obtained in an arterial segment cannot be extrapolated to other segments in the same artery tree. This is because pressure wave components (backflow and reflection) change with age, gender and the presence of risk factors such as diabetes, smoking, dyslipidemia and others.

The angiotensin II receptor blocker, losartan, in comparison to the beta-blocker, atenolol, showed greater efficacy in reducing cardiovascular events and lesions to target organs in the LIFE study despite of similar reductions in brachial blood pressure. The results of some randomized clinical trials such as the one by Klingbeil et al confirmed significant reductions in AIx with valsartan as monotherapy compared with a thiazide diuretic or placebo. When valsartan was compared to captopril both reduced the AIx and PWV similarly [27].

Dakhan et al compared the effects of atenolol versus eprosar$\tan$ (BRA) on central and peripheral hemodynamics in a doubleblind randomized crossover trial. The results indicated that for the same reduction in brachial pressure, there was a greater reduction in the central pressure and reduction in wave reflection [28].

The EXPLOR trial that compared the association of valsartan and amlodipine with amlodipine and atenolol showed similar reductions in brachial artery pressure with both combination therapies. The PWV and AIx showed significantly higher decreases in the valsartan/amlodipine group than in the amlodipine/atenolol group, and thus this association had a better effect on the central hemodynamics [29].

The results of the Conduit Artery Function Evaluation (CAFE) trial [18] corroborate the findings of this study. The authors of that study showed that the group that took a thiazide diuretic with atenolol had a lower reduction in CBP than the group treated with angiotensin converting enzyme inhibitors. This important clinical finding is the basis of current guidelines for the treatment of hypertension and reduction of cardiovascular risk. There were reductions in SBP, DBP and MAP with treatment, which impeded amplification of the central pressure wave and reduced the CBP.

Shimizu et al highlight the importance of using the mean $\mathrm{BP}$ as an adjunct measure of antihypertensive therapy. The authors showed an association between the reduction in CBP

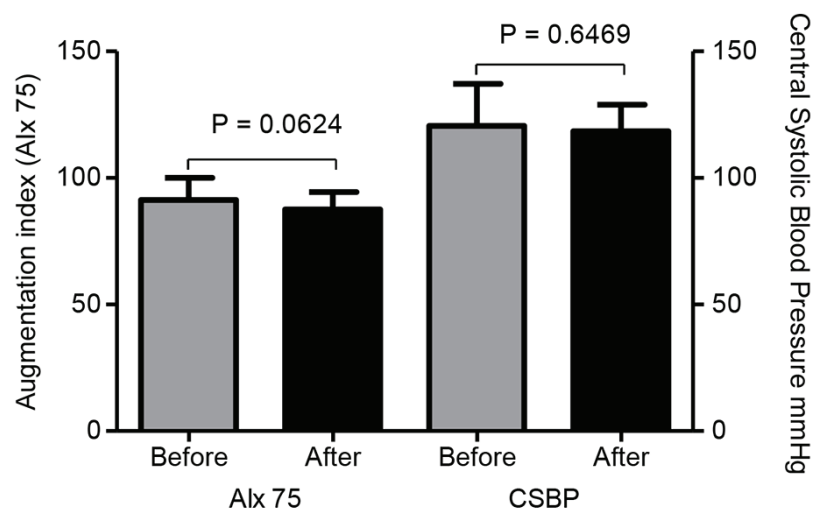

Figure 3. Central systolic blood pressure and augmentation index at baseline and after treatment with losartan $50 \mathrm{mg}$ (b.i.d.) in the HTN-1 patients. CSBP: central systolic blood pressure; Alx 75: augmentation index corrected for average heart rate of 75 beats/min; HTN-1: stage 1 hypertension.

and decreased urinary albumin excretion (a marker of endothelial dysfunction) and left ventricular mass index (target organ damage) in hypertensive patients treated with antihypertensive drugs [30].

The relevance and potential of this method lies in the importance of the difference found between the CSBP and peripheral brachial BP in all age groups as demonstrated by McEniery et al in the Anglo-Cardiff Collaborative Trial II [21]. Additionally, the influence of antihypertensive therapy on the behavior of CSBP of HTN-1 patients cannot be excluded. However, new perspectives of structural and functional hemodynamics are needed to confirm the findings presented.

Our results reinforce the importance of early pharmacological interventions in HTN-1 patients because besides reducing peripheral $\mathrm{BP}$, low doses of chlorthalidone added to amiloride had a beneficial effect within 6 months of therapy.

\section{Conclusion}

From the sixth month, treatment with chlorthalidone $25 \mathrm{mg}$ added to amiloride $5 \mathrm{mg}$ (q.d.), but not losartan $50 \mathrm{mg}$ (b.i.d.), reduces the CSBP and the AIx in adults with HTN-1.

\section{Financial Disclosure}

None.

\section{References}

1. Nwankwo T, Yoon SS, Burt V, Gu Q. Hypertension among adults in the United States: National Health and Nutrition Examination Survey, 2011-2012. NCHS Data Brief. 2013;(133):1-8.

2. Tibazarwa KB, Damasceno AA. Hypertension in developing countries. Can J Cardiol. 2014;30(5):527-533. 
3. Chobanian AV, Bakris GL, Black HR, Cushman WC, Green LA, Izzo JL, Jr., Jones DW, et al. Seventh report of the Joint National Committee on Prevention, Detection, Evaluation, and Treatment of High Blood Pressure. Hypertension. 2003;42(6):1206-1252.

4. Durier S, Fassot C, Laurent S, Boutouyrie P, Couetil JP, Fine E, Lacolley P, et al. Physiological genomics of human arteries: quantitative relationship between gene expression and arterial stiffness. Circulation. 2003;108(15):1845-1851.

5. Hamilton PK, Lockhart CJ, Quinn CE, McVeigh GE. Arterial stiffness: clinical relevance, measurement and treatment. Clin Sci (Lond). 2007;113(4):157-170.

6. Soiza RL, Davie MM, Williams DJ. Use of the augmentation index to predict short-term outcome after acute ischemic stroke. Am J Hypertens. 2010;23(7):737-742.

7. Safar H, Mourad JJ, Safar M, Blacher J. Aortic pulse wave velocity, an independent marker of cardiovascular risk. Arch Mal Coeur Vaiss. 2002;95(12):1215-1218.

8. Laurent S, Katsahian S, Fassot C, Tropeano AI, Gautier I, Laloux B, Boutouyrie P. Aortic stiffness is an independent predictor of fatal stroke in essential hypertension. Stroke. 2003;34(5):1203-1206.

9. Asmar R, Topouchian J, Pannier B, Benetos A, Safar M. Pulse wave velocity as endpoint in large-scale intervention trial. The Complior study. Scientific, Quality Control, Coordination and Investigation Committees of the Complior Study. J Hypertens. 2001;19(4):813-818.

10. Troen BR. The biology of aging. Mt Sinai J Med. 2003;70(1):3-22.

11. Podolec P, Kopec G, Podolec J, Wilkolek P, Krochin M, Rubis P, Cwynar M, et al. Aortic pulse wave velocity and carotid-femoral pulse wave velocity: similarities and discrepancies. Hypertens Res. 2007;30(12):1151-1158.

12. Figueiredo VN, Yugar-Toledo JC, Martins LC, Martins LB, de Faria AP, de Haro Moraes C, Sierra C, et al. Vascular stiffness and endothelial dysfunction: Correlations at different levels of blood pressure. Blood Press. 2012;21(1):31-38.

13. Van Bortel LM, Balkestein EJ, van der Heijden-Spek JJ, Vanmolkot FH, Staessen JA, Kragten JA, Vredeveld JW, et al. Non-invasive assessment of local arterial pulse pressure: comparison of applanation tonometry and echotracking. J Hypertens. 2001;19(6):1037-1044.

14. Nelson MR, Stepanek J, Cevette M, Covalciuc M, Hurst RT, Tajik AJ. Noninvasive measurement of central vascular pressures with arterial tonometry: clinical revival of the pulse pressure waveform? Mayo Clin Proc. 2010;85(5):460-472.

15. Gupta R, Guptha S. Strategies for initial management of hypertension. Indian J Med Res. 2010;132:531-542.

16. Dias Eda M, Giollo LT, Jr., Martinelli DD, Mazeti C, Junior HM, Vilela-Martin JF, Yugar-Toledo JC. Carotid intima-media thickness is associated with cognitive deficiency in hypertensive patients with elevated central systolic blood pressure. Cardiovasc Ultrasound. 2012;10:41.

17. Joffres M, Falaschetti E, Gillespie C, Robitaille C, Loustalot F, Poulter N, McAlister FA, et al. Hypertension prevalence, awareness, treatment and control in national surveys from England, the USA and Canada, and correlation with stroke and ischaemic heart disease mortality: a cross-sectional study. BMJ Open. 2013;3(8):e003423.

18. Williams B, Lacy PS, Thom SM, Cruickshank K, Stanton A, Collier D, Hughes AD, et al. Differential impact of blood pressure-lowering drugs on central aortic pressure and clinical outcomes: principal results of the Conduit Artery Function Evaluation (CAFE) study. Circulation. 2006;113(9):1213-1225.

19. McEniery CM, Cockcroft JR, Roman MJ, Franklin SS, Wilkinson IB. Central blood pressure: current evidence and clinical importance. Eur Heart J. 2014;35(26):17191725.

20. Al-Solaiman Y, Jesri A, Mountford WK, Lackland DT, Zhao Y, Egan BM. DASH lowers blood pressure in obese hypertensives beyond potassium, magnesium and fibre. $\mathrm{J}$ Hum Hypertens. 2010;24(4):237-246.

21. McEniery CM, Yasmin, McDonnell B, Munnery M, Wallace SM, Rowe CV, Cockcroft JR, et al. Central pressure: variability and impact of cardiovascular risk factors: the Anglo-Cardiff Collaborative Trial II. Hypertension. 2008;51(6):1476-1482.

22. Trinder P. Determination of blood glucose using 4-amino phenazone as oxygen acceptor. J Clin Pathol. 1969;22(2):246.

23. Bucolo G, David H. Quantitative determination of serum triglycerides by the use of enzymes. Clin Chem. $1973 ; 19(5): 476-482$.

24. Allain CC, Poon LS, Chan CS, Richmond W, Fu PC. Enzymatic determination of total serum cholesterol. Clin Chem. 1974;20(4):470-475.

25. Friedewald WT, Levy RI, Fredrickson DS. Estimation of the concentration of low-density lipoprotein cholesterol in plasma, without use of the preparative ultracentrifuge. Clin Chem. 1972;18(6):499-502.

26. Levey AS, Greene T, Beck GJ, Caggiula AW, Kusek JW, Hunsicker LG, Klahr S. Dietary protein restriction and the progression of chronic renal disease: what have all of the results of the MDRD study shown? Modification of Diet in Renal Disease Study group. J Am Soc Nephrol. 1999;10(11):2426-2439.

27. Klingbeil AU, John S, Schneider MP, Jacobi J, Weidinger G, Schmieder RE. AT1-receptor blockade improves augmentation index: a double-blind, randomized, controlled study. J Hypertens. 2002;20(12):2423-2428.

28. Dhakam Z, McEniery CM, Yasmin, Cockcroft JR, Brown MJ, Wilkinson IB. Atenolol and eprosartan: differential effects on central blood pressure and aortic pulse wave velocity. Am J Hypertens. 2006;19(2):214-219.

29. Boutouyrie P, Achouba A, Trunet P, Laurent S. Amlodipine-valsartan combination decreases central systolic blood pressure more effectively than the amlodipine-atenolol combination: the EXPLOR study. Hypertension. 2010;55(6):1314-1322.

30. Shimizu M, Hoshide S, Ishikawa J, Yano Y, Eguchi K, Kario K. Correlation of Central Blood Pressure to Hypertensive Target Organ Damages During Antihypertensive Treatment: The J-TOP Study. Am J Hypertens. 2015;28(8):980-986. 